\title{
Alloy: A New Technology for Software Modelling
}

\author{
Daniel Jackson
}

Software Design Group, Laboratory for Computer Science

Department of Electrical Engineering \& Computer Science, MIT

200 Technology Square, Cambridge, Mass 02139, USA

\begin{abstract}
Alloy is a lightweight language for software modelling. It's designed to be flexible and expressive, and yet amenable to fully automatic simulation and checking. At its core, Alloy is a simple first order logic extended with relational operators. A simple structuring mechanism allows Alloy to be used in a variety of idioms, and supports incremental construction of models. Alloy is analyzed by translation to SAT. The current version of the tool uses the Chaff and Berkmin solvers; these are powerful enough to handle a search space of $2^{100}$ or more. Alloy has been applied to problems from very different domains, from checking the conventions of Microsoft COM to debugging the design of a name server. Most recently, we have used it to check distributed algorithms that are designed for arbitrary topologies. We are also investigating the use of Alloy to analyze object-oriented code.
\end{abstract}

